\title{
OFERTA DE ESTUDIOS DE INSTITUCIONES ALEMANAS DE EDUCACIÓN SUPERIOR EN EL CONTEXTO DE LA COOPERACIÓN INTERNACIONAL
}

Desde principios de los noventa, la "exportación" de servicios educacionales ha adquirido importancia. Inglaterra, Australia y Estados Unidos de Norteamérica, especialmente, se posicionan con ofertas de estudios en el mercado internacional de la educación.

Actualmente, ya se han inscrito más de 140.000 y sobre 42.000 alumnos en los programas de estudios británicos y australianos, respectivamente. Estos países saben, desde hace mucho tiempo, que la educación es un importante mercado en el que vale la pena invertir, y cada institución de educación superior asocia claros objetivos económicos a la exportación de educación. En Australia esta oferta se ha convertido en un factor económico de consideración.

Las instituciones alemanas de educación superior (IAES) que desean implementar ofertas de estudios en el extranjero enfrentan, en varios aspectos, un especial desafío:

1. Se encuentran en un mercado donde ya están bien establecidos otros oferentes, principalmente en importantes regiones del sudeste asiático elegidas como objetivo.

2. Esta nueva proyección exige una función empresarial que las IAES, predominantemente estatales, aún no han tenido que asumir en la misma medida en que lo ha hecho la mayoría de sus competidoras internacionales. Sin embargo, debido a su financiamiento mayoritariamente estatal, hasta ahora no habían tenido realmente la necesidad de actuar conforme a criterios empresariales y de competir con otros oferentes.

3. Otra desventaja frente a las citadas competidoras es el idioma. Resulta evidente que los países de habla inglesa tienen una ventaja real de posicionamiento. 
El programa de fomento "Oferta de estudios en el extranjero de instituciones alemanas de educación superior" del Servicio Alemán de Intercambio Académico (DAAD) permite que, por primera vez, estas organizaciones realicen y sometan a prueba una amplia oferta de estudios. Las nuevas carreras, las facultades y las IAES se destacan por el alto grado de compromiso de los profesores universitarios alemanes y la estrecha cooperación con el sector económico. Mediante este programa, se fortalece su presencia en el mercado internacional de la educación.

\section{1. "Exportación" de servicios educacionales}

\subsection{Programa "Oferta de estudios en el extranjero de las instituciones alemanas de educación superior" y sus objetivos}

Al principio las IAES se presentaban con sus propios programas de estudios sólo en algunos lugares elegidos, principalmente en el este y sudeste de Europa y también en Asia. Estas iniciativas, respaldadas principalmente por el DAAD, se guiaban por consideraciones políticas y político-universitarias. Algunos proyectos son, por ejemplo, la Andrássy-Universität de habla alemana en Budapest, la Facultad de Administración de Empresas e Ingeniería Comercial, con estudios en idioma alemán en Sofía, y la escuela superior chino-alemana Deutsches Hochschulkolleg en Shangai.

El programa del DAAD "Ofertas de estudios en el extranjero de instituciones alemanas de educación superior" fue introducido en 2001. Sus objetivos son:

- Brindar a las IAES la posibilidad de competir con oferentes de otros países en el mercado internacional de la educación.

- Probar distintos modelos y formas de organización para nuevas ofertas de estudios en el extranjero: desde una sola carrera, en el marco de actividades de cooperación, facultades completas, con diversas ofertas de estudios, hasta instituciones filiales o de educación superior independientes en el extranjero. 
- Lograr un mejor posicionamiento de las ofertas alemanas de estudios en el mercado internacional de la educación. Se fomenta el establecimiento de IAES competitivas con una planificación conforme a criterios empresariales. Ello les brinda la posibilidad de captar alumnos de doctorado altamente calificados y de desarrollar nuevas actividades de cooperación con universidades extranjeras asociadas. Se recomienda enfáticamente la cooperación con empresas del sector económico, dentro y fuera del país.

En lo posible, estas ofertas deben conducir a un grado o título académico alemán o reconocido internacionalmente, y deben, sobre todo, estar sometidas a controles de calidad permanentes de la institución "exportadora" de educación superior.

La implementación de oferta alemana de estudios otorga numerosas ventajas a las entidades extranjeras asociadas con las IAES:

- Las nuevas ofertas complementan en un alto nivel sus propios programas de estudios.

- Las nuevas materias se ofrecen en el país de la entidad extranjera.

- Los grados o títulos académicos alemanes, o grados o títulos dobles, incrementan el atractivo de la universidad asociada.

- Se activa y fortalece la cooperación y el intercambio en el ámbito científico y, por consiguiente, se incrementa el prestigio internacional de la universidad extranjera.

\subsection{Cifras y datos}

Actualmente se patrocinan 29 proyectos en el marco del Programa. Diez corresponden a universidades, seis a universidades técnicas, once a instituciones de educación técnica superior y dos a instituciones de educación superior de Música y Arte. 
Gráfico 1. Distribución por instituciones de educación superior

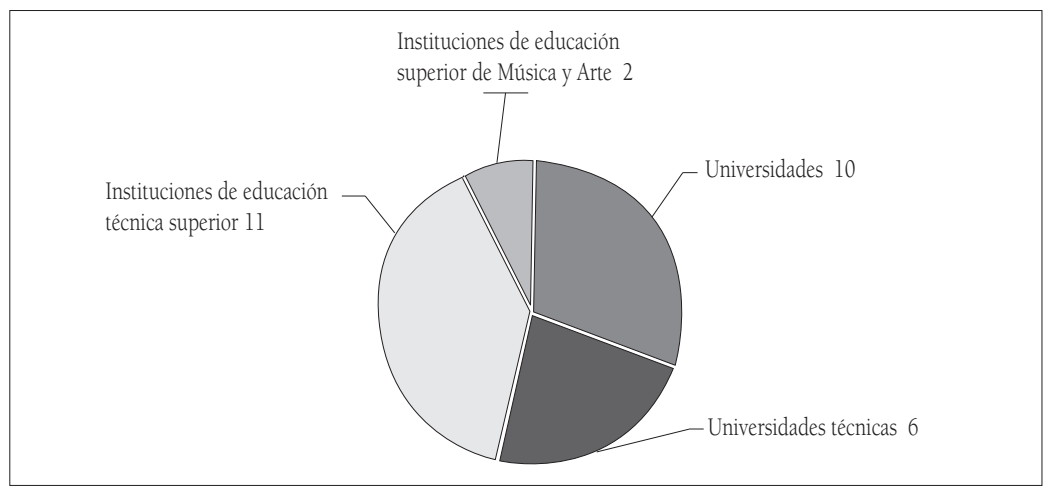

Gráfico 2. Áreas de especialización de las ofertas de estudios

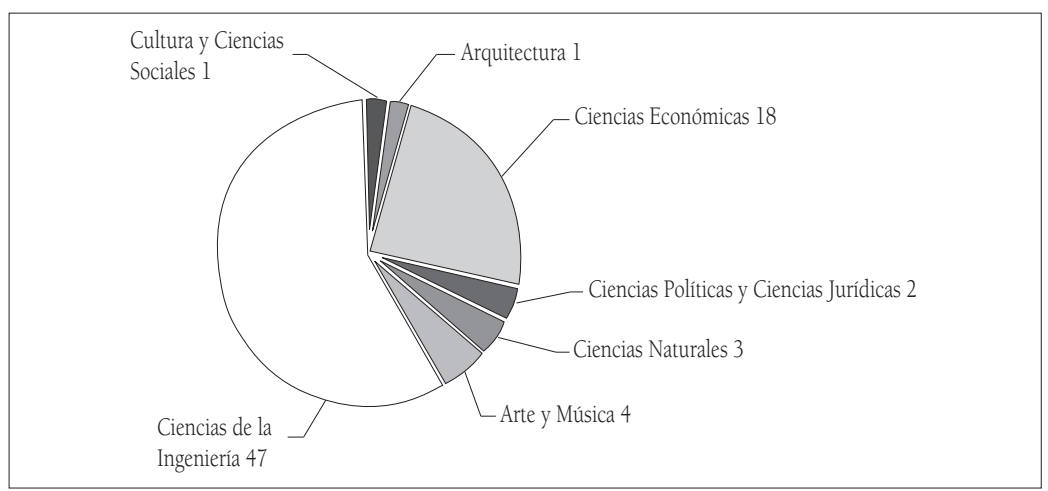

En 2005 se ofreció un total de 76 carreras regulares en el marco de estos proyectos. Entre las especialidades predominan las Ciencias de la ingeniería con 47 ofertas de estudios; 18 corresponden a Ciencias económicas, cuatro a las especialidades de Arte y Música, tres a Ciencias naturales y las ofertas restantes se distribuyen entre las demás áreas de especialización.

La región más importante elegida como objetivo es Asia, donde se desarrollan 14 de 29 proyectos. En América Latina también se dio inicio a cuatro proyectos de cooperación con el objetivo de desarrollar ofertas de estudios conjuntas con las respectivas universidades asociadas. 
En la primavera de 2005 ya se habían inscrito 5.300 alumnos en los programas de estudios promocionados; en 2008 serán más de 13.000 alumnos sólo en los 29 proyectos. Si se suman las ofertas de estudios fomentadas por razones político-culturales en el este y sudeste de Europa y en China, y las de instituciones que han desarrollado ofertas de estudios sin el patrocinio del DAAD, tenemos que más de 6.500 jóvenes estudian carreras alemanas en el extranjero.

\section{Los distintos modelos}

\subsection{Modelos de exportación de ofertas de estudios en Alemania}

La motivación de las IAES para presentar sus ofertas de estudios en el extranjero no está principalmente en la creación de nuevas áreas comerciales rentables. Con escasas excepciones, los proyectos se enmarcan más bien dentro del concepto "sin fines de lucro", es decir, los aranceles recaudados y demás recursos aportados por terceros se destinan principalmente a cubrir los costos corrientes. Para muchas de estas instituciones, el objetivo es tan sólo no quedar con saldo negativo tras el financiamiento inicial y poder seguir ofreciendo estudios sin ayuda estatal. Muchos proyectos tienen un trasfondo asociado a políticas de desarrollo y se basan en la cooperación de años de científicos y facultades participantes con las universidades asociadas en el extranjero.

En total, pueden distinguirse cuatro grupos de ofertas de estudios en el extranjero:

a) Implementación de programas de estudios fundada en razones de política cultural y política exterior. Aquí cabe citar, en primer lugar, los programas en idioma alemán en Europa Oriental, fomentados desde principios de los años noventa mediante un programa especial del DAAD. Se han puesto en marcha alrededor de 20 de estos programas, en los que participan actualmente unas 750 personas. Otros ejemplos son la citada Andrássy-Universität en Budapest y la Facultad de Administración de Empresas e 
Ingeniería Comercial con estudios en idioma alemán en Sofía. En general, estos proyectos requieren un respaldo financiero permanente de la parte alemana.

b) Participación en la creación de instituciones alemanas de educación superior en el extranjero. El ejemplo más conocido es, por cierto, la Universidad Alemana en El Cairo (German University in Cairo, GUC), una universidad egipcia privada recientemente fundada. Asociadas principales de la GUC son las universidades alemanas de Stuttgart y Ulm, que tienen una participación determinante en su diseño conceptual y en el desarrollo de las mallas curriculares. En el consejo superior de la universidad participan diversos miembros alemanes y su cuerpo docente tiene alto porcentaje de profesores de esta nacionalidad.

Otros ejemplos son la Deutsch-Jordanische Hochschule (Universidad Jordano-Alemana, GJU), en proceso de desarrollo en Ammán, y la Chinesisch-Deutsche Hochschule für Angewandte Wissenschaften (Universidad Chino-Alemana de Ciencias Aplicadas, CDHAW) en Shangai, con la participación de grandes conglomerados de universidades técnicas alemanas.

Se proyecta impartir programas de estudios conducentes al grado académico alemán y extranjero de bachiller o magíster. Para el mediano plazo, también se piensa integrar actividades de investigación de manera análoga al sistema universitario alemán. Las clases se imparten en inglés, pero todos los alumnos también aprenden alemán durante la carrera. Parte de los estudios debe realizarse de manera obligatoria o voluntaria en Alemania.

En esta clase de universidades, la construcción y el financiamiento de la infraestructura y de las operaciones corrientes es de responsabilidad de la asociada extranjera. Estos costos se garantizan substancialmente con participación del gobierno extranjero o de inversionistas privados, y la recaudación de aranceles, entre otros medios. Por consiguiente, el aporte financiero alemán es principalmente un financiamiento inicial para sus gastos de coordinación. Debido a la gran difusión e 
importancia de estos grandes proyectos en el marco de las políticas universitarias, el apoyo y la participación a largo plazo del sector público también dependen fuertemente de la política cultural y exterior.

c) Comercialización de ofertas de estudios alemanas en el marco de la cooperación con una universidad extranjera. La mayoría de las carreras del programa se ofrecen en este marco. A menudo, los programas de estudio complementan la oferta de la universidad asociada con materias que hasta la fecha no se incluían o no se ofrecían al nivel correspondiente. Las mallas curriculares se definen en conjunto con la universidad extranjera y, generalmente, conducen a un doble grado o título académico. Frecuentemente, se prevén estadías obligatorias o facultativas para fines de estudio en Alemania.

En general, ello se basa en un convenio de cooperación entre la institución alemana de educación superior y la extranjera. Los ingresos obtenidos por concepto de actividades de investigación y aranceles de estudios se distribuyen entre las asociadas y se destinan principalmente a cubrir los gastos corrientes (costos de movilización, gastos de cooperación, honorarios, entre otros). Un ejemplo de esta clase de joint-venture es el programa de estudios Nachhaltige Siedlungsund Infrastrukturplanung (Planificación sustentable de urbanización e infraestructura) de la Universidad Técnica de Braunschweig, que se está llevando a cabo en conjunto con la Pontificia Universidad Católica de Río de Janeiro. La estrecha cooperación con asociados externos confiere un carácter especial al programa de estudios: el apoyo de éstos consiste en ofrecer lugares de práctica, becas y docentes para determinados modelos de enseñanza. La estrecha colaboración garantiza una alta orientación a la práctica y a la aplicación. Hasta ahora, las ofertas de estudios existentes en la universidad brasileña no contemplan esta proyección especial.

Una variante de este modelo apunta, por ejemplo, a ofrecer estudios básicos o el bachillerato en el extranjero con las mismas 
materias (y clases adicionales de idioma) que en Alemania. La realización queda esencialmente a cargo de la universidad asociada. Luego, la totalidad o parte de los alumnos viaja a Alemania para realizar los estudios principales o los conducentes al grado de magíster. Obtienen entonces el grado académico en ambas universidades.

d) Establecimiento de agencias de IAES en el extranjero. Algunas instituciones han establecido representaciones o agencias como sociedades de responsabilidad limitada en el extranjero. En esta categoría puede incluirse el Heidelberg Center de la Universidad de Heidelberg en Santiago de Chile, que sirve de plataforma para las ofertas de estudios de distintas instituciones de educación superior de Baden-Wurtemberg. También en este caso existe una estrecha asociación con universidades locales, cuya infraestructura se utiliza en parte en forma conjunta.

\subsection{Características de las ofertas alemanas de estudios en el extranjero}

Que las entidades creadas en el extranjero tengan acogida en el mercado depende de la calidad de las ofertas de estudios y de su aceptación en el mercado laboral respectivo. Estas ofertas se basan en un currículo desarrollado por las IAES y conducen generalmente a un grado o título académico reconocido.

En los 29 proyectos del programa "Ofertas de estudios en el extranjero de las instituciones alemanas de educación superior" se ofrecen, en total, según datos de las universidades (2005), 76 programas de estudios regulares o grados/títulos académicos.

Se trata de 36 programas conducentes al grado de bachiller, 37 que confieren el grado de magíster, y tres diplomados. En 2008, sólo los 29 proyectos patrocinados a la fecha ofrecerán alrededor de 100 programas.

Se cobran aranceles en casi todas las ofertas de estudios. El monto promedio es de aproximadamente 2.100 euros por semestre; 
en siete el arancel es inferior a 1.000 euros, en once está entre 1.000 y 2.000 euros y en otros once es superior a 2.000 .

\section{Grados o títulos académicos}

En la mayoría de las carreras, los alumnos podrán obtener tanto el título o grado académico alemán como el extranjero, o bien un diploma doble. En algunos casos, se exige cursar una parte de los estudios en Alemania para obtener el título o grado académico alemán. Junto con el reconocimiento del título o grado académico, la acreditación también es un elemento de garantía de calidad a la que aspira la mayoría de los proyectos.

\section{Participación de profesores universitarios}

Una característica especial de los proyectos patrocinados es la comprometida participación de los profesores universitarios alemanes en los programas de estudios y, por consiguiente, la estrecha relación con la universidad matriz. Cerca del $40 \%$ de las actividades académicas están a cargo de profesores universitarios alemanes y, a menudo, de profesores universitarios extranjeros que estudiaron en Alemania. Esta es una característica esencial de la calidad de las ofertas, las que en este sentido se diferencian claramente de las de Gran Bretaña o Australia. Muchas entidades derivadas de las existentes unen la oferta de estos programas con una estrecha cooperación en investigación.

En 34 carreras, cursar una parte de los estudios en Alemania es -además- un componente obligatorio del currículo; en casi todos los programas de estudios se establece opcionalmente una estadía en Alemania.

\section{Clases de idioma}

Independientemente del tipo de proyecto, se adoptaron diversas normas en relación con el idioma en que se imparten las clases: de las 76 ofertas, 32 se imparten en inglés, cuatro en alemán, tres en una combinación de alemán e inglés, 25 en una combinación de alemán 
y/o inglés con el idioma del país, y 12 en una combinación de los tres idiomas. Por consiguiente, el inglés es el idioma predominante, aunque en 25 de estos 40 programas en idiomas mixtos también se dictan clases en alemán.

El DAAD no determina el idioma en que se imparten las clases, sino que éste se establece de manera práctica con la asociada en la cooperación, según la situación. En cada caso debe ofrecerse simultáneamente el idioma alemán. Esta exigencia se cumple en casi todos los proyectos.

\section{Estrecha cooperación con el sector económico}

En muchos proyectos, la implementación de ofertas de estudios en el extranjero ha llevado a estrechas actividades de cooperación con el sector económico e industrial, dentro y fuera del país: deben ofrecerse lugares de práctica atractivos a los alumnos y perspectivas profesionales a los graduados. De este sector se obtuvieron paquetes de becas, fondos para la investigación e incluso cátedras de la institución. En algunos programas se cuenta también con la participación de representantes de la industria en el desarrollo de la malla curricular y en el aprendizaje.

\section{Tareas centrales en la materialización de los proyectos}

Con el programa se penetra en territorio inexplorado en cuanto a política universitaria. Las IAES partícipes se mueven en un terreno no acostumbrado. Proyectan nuevas áreas de negocios que, en unos pocos años, se autosustenten o que, incluso, sean rentables, es decir, generalmente recaudan aranceles y buscan activamente el respaldo y el patrocinio de la industria. A diferencia de como había sido hasta ahora en Alemania, normalmente ellas mismas pueden elegir a sus futuros alumnos, pero también deben encargarse de la comercialización y de una activa garantía de calidad. Desde el punto de vista del DAAD, al desarrollar ofertas de estudios en el extranjero han surgido algunas tareas centrales, de especial importancia en lo que 
se refiere a la planificación de futuros proyectos o para la continuidad de los existentes. Aquí se citan principalmente áreas en las que se han originado distintas vías de solución.

\subsection{Identificación de ofertas de estudios factibles de comercializar}

Como se mencionó anteriormente, la idea de crear una entidad derivada de una existente surge -en la mayoría de los casos-de las actividades de cooperación entre profesores universitarios o facultades individuales y asociados extranjeros. Antes de la materialización, es necesario realizar los estudios de mercado correspondientes para verificar la sustentabilidad de los proyectos planificados conforme a criterios empresariales. También podría pensarse en el camino inverso, aunque hasta ahora poco practicado: la institución de educación superior identifica una oferta de estudios de excelencia y especialmente apropiada, y busca un lugar adecuado para presentarla y comercializarla.

\subsection{Implementación de actividades de cooperación sustentables y decisión respecto del régimen jurídico}

La mayoría de los proyectos patrocinados realizan sus ofertas de estudios como joint-ventures, en conjunto con universidades asociadas en el extranjero. Incluso cuando estos proyectos se basan en actividades de cooperación de larga trayectoria y probada eficacia, las expectativas frente a ellos son frecuentemente muy distintas: pueden modificarse durante su implementación o no cumplirse. Es necesario, por tanto, convenir con mucha anticipación las disposiciones contractuales. Estas comprenden, además de la decisión sobre asuntos curriculares, un plan mancomunado de costos y financiamiento, acuerdos sobre la utilización de aranceles y otros ingresos, cláusulas respecto de las obligaciones de los socios en caso de que no se logren las cifras esperadas, acuerdos sobre la utilización de los espacios en que se imparten clases, laboratorios y otras instalaciones, cláusulas respecto de la proporción planificada de docentes alemanes en la oferta de estudios, participación en los exámenes, participación en las juntas rectoras, entre otras. 
Si bien dichos acuerdos no deben considerarse como algo estático, proporcionan la base para el entendimiento ante posibles problemas.

Las instituciones de educación superior que se establecen en el extranjero como empresas independientes (por ejemplo, como sociedades de responsabilidad limitada) deberían analizar previamente la situación del mercado y los requisitos jurídicos y legales básicos. Un muy buen conocimiento del país es una condición indispensable. También aquí parece ventajosa una estrecha cooperación con una universidad asociada, con el fin de mantener bajos los costos de inversión y beneficiarse de su experiencia y prestigio.

\subsection{Garantía de la enseñanza mediante un número suficiente de docentes alemanes calificados}

Esta garantía sigue siendo, a largo plazo, un asunto medular y es muy importante para la sustentabilidad de los proyectos. Para muchos profesores universitarios la etapa inicial tiene el estímulo de lo nuevo, lo que los lleva a asumir obligaciones docentes en el extranjero; sin embargo, esto no se da necesariamente en el caso de desempeños prolongados. En el largo plazo, la movilización, los honorarios, los costos de atención, entre otros aspectos, deben financiarse con los ingresos del proyecto. Algunas universidades tratan de contratar como profesores a graduados propios pero que provienen del país elegido como objetivo, por ejemplo, tras obtener con éxito el grado de doctor. Ello presenta problemas, especialmente cuando los sueldos del sector industrial en los respectivos países son evidentemente más altos que los pagados a los profesores universitarios.

Los costos de personal podrían reducirse en el largo plazo mediante la incorporación de módulos de aprendizaje vía electrónica y del aprendizaje a distancia.

\subsection{Grados o títulos académicos y reconocimiento}

Casi todos los proyectos han desarrollado ofertas de estudios en que se obtienen los títulos o grados habituales. Para garantizar su calidad 
es conveniente conferir un grado o título alemán, un diploma doble y/o un grado o título reconocido internacionalmente. Especialmente allí donde las ofertas alemanas compiten con otras, cobrará creciente importancia el reconocimiento internacional. Para la creación de nuevas ofertas en el país elegido como objetivo es importante determinar si los títulos o grados serán reconocidos, en qué momento y cuál será la institución encargada de ello. En el caso de los nuevos programas de estudios también hay que considerar que los alumnos que no logren el rendimiento exigido puedan ser aceptados en el sistema regular extranjero.

\subsection{Garantía permanente de clases de alemán como idioma} de enseñanza o como oferta complementaria

La relación con Alemania y, por consiguiente, las clases de alemán son un elemento esencial de la oferta de estudios. Aunque el idioma de enseñanza se haya elegido conforme a criterios más bien pragmáticos, debe garantizarse al menos la enseñanza de alemán con clases paralelas a los estudios. Este es el caso, en particular, cuando parte de los estudios o las prácticas deben realizarse en Alemania o cuando se desea que los mejores alumnos titulados realicen estudios de perfeccionamiento en este país.

\subsection{Seguridad financiera del proyecto total}

En muchos proyectos, la seguridad financiera en el largo plazo aún no está lo suficientemente dilucidada. Si bien se ha hecho lo correcto al recaudar los aranceles de estudio y al obtener paquetes de becas del sector económico, surge la duda de si esos proyectos podrán financiarse sólo con estos aranceles. Aun cuando pueda esperarse que un número suficiente de proyectos logre independencia financiera en el largo plazo, según las experiencias del primer período de patrocinio de este programa debe calcularse una etapa de puesta en marcha de tres a seis años hasta poder trabajar cubriendo los costos.

En algunos proyectos no pudo lograrse el número previsto de alumnos. Ya en la fase de planificación se requiere un concepto 
de marketing para la implementación de ofertas de estudios. Deben considerarse las capacidades y los recursos necesarios para publicidad, presencia en internet, participación en ferias, viajes para dictar conferencias en otras universidades, entre otros recursos. Según la oferta, la estrategia de marketing debe referirse también a otros países de la región respectiva.

La implementación continua de actividades de cooperación con empresas internacionales en la región también resulta indispensable para muchos proyectos. Debe analizarse la forma de generar ingresos adicionales mediante encargos de trabajos de investigación u ofertas de estudios de perfeccionamiento.

\subsection{Establecimiento de una gestión continuada del proyecto por el lado alemán}

Si bien el entusiasmo y el compromiso de los científicos son elementos esenciales del proyecto, no reemplazan en forma permanente una gestión profesional de éste. La gestión del proyecto contempla, entre otros, la coordinación administrativa y financiera, la obtención de financiamiento de terceros, los contactos con el sector industrial, el marketing y la publicidad. Especialmente cuando se han planificado varias ofertas de estudios, se requiere un interlocutor permanente en Alemania y en el país elegido como objetivo. Estas tareas no las pueden realizar paralelamente a sus labores los profesores universitarios de estadía transitoria.

\subsection{Incorporación del proyecto a un concepto integral de internacionalización de la IAES}

Para que los proyectos sean sustentables, es muy importante que estén administrativa y estratégicamente arraigados en la propia universidad. La "asistencia" permanente a los proyectos debe organizarse para el largo plazo. Ello se refiere al reconocimiento de los programas de estudios, controles de calidad, garantía de enseñanza, trabajo de alumnos y respaldo financiero. Deben crearse estructuras que dentro de algunos años garanticen todavía el cumplimiento adecuado de las obligaciones contraídas en el extranjero. 
Muchos proyectos han logrado al poco tiempo una enorme importancia en cuanto a política universitaria y un significado que va mucho más allá de las asociaciones universitarias normales. Especialmente allí donde las ofertas de estudios están expuestas a una fuerte competencia internacional o donde, por la magnitud del proyecto, tienen difusión suprarregional, se registra y se analiza con precisión el éxito y el fracaso. Así, un fracaso no sólo tiene un impacto negativo en el proyecto en sí, sino que puede dañar además el prestigio internacional de la institución de educación superior, del DAAD o de Alemania como lugar para estudiar. Por ello, es muy importante también el activo respaldo por parte de la rectoría de la universidad respectiva.

\section{Territorio inexplorado en el ámbito de la política universitaria}

Con la implementación de las ofertas de estudios en el extranjero se ingresa a territorio inexplorado desde el punto de vista de la política universitaria. Mediante la creación de nuevas instituciones derivadas de las existentes, se han abierto a éstas nuevos campos en el marco de la internacionalización y también la oportunidad de probar, en el mercado internacional, ofertas de estudios planificadas conforme a criterios empresariales. La vinculación entre enseñanza e investigación, típica de la cultura docente alemana, y la estrecha cooperación con el sector económico son características destacadas de las ofertas de estudios en el extranjero.

Mucho de lo que se prueba con la implementación de estas ofertas de estudios no constituye una operación habitual para la mayoría de las instituciones de educación superior en Alemania. La planificación de las ofertas de estudios conforme a criterios empresariales, la recaudación de aranceles de estudio, la selección de los alumnos orientada a la calidad, la obtención de fondos y la estrecha cooperación con el sector económico orientado a la exportación exigen un gran compromiso y esfuerzo para emprender la hazaña. Cabe considerar que, en una u otra empresa, habrá que 
pagar aún un precio alto para lograr que el saldo no sea negativo. Sin embargo, los primeros resultados son más que alentadores. El número de alumnos inscritos aumenta continuamente; también se registran considerables beneficios intangibles: el aumento del prestigio y de la internacionalidad, la obtención de nuevos socios y la intensificación del trabajo conjunto con el sector económico orientado al comercio exterior convierten a la exportación de educación en un campo de acción de gran porvenir para las IAES.

Recibido: 24 de mayo de 2007 Aceptado: 5 de junio de 2007 